Modeling, Identification and Control, Vol. 29, No. 2, 2008, pp. 67-75

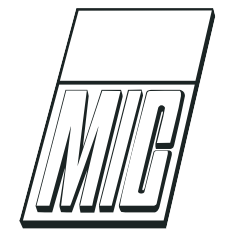

\title{
Optimizing Adaptive Control Allocation With Actuator Dynamics
}

\author{
Johannes Tjønnås and Tor Arne Johansen
}

Department of Engineering Cybernetics, Norwegian University of Science and Technology, N-7491 Trondheim, Norway.

\begin{abstract}
In this work we address the optimizing control allocation problem for an over-actuated nonlinear timevarying system with actuator dynamic where parameters affine in the actuator and effector model may be assumed unknown. Instead of optimizing the control allocation at each time instant, a dynamic approach is considered by constructing actuator reference update-laws that represent an asymptotically optimal allocation search. By using Lyapunov analysis for cascaded set-stable systems, uniform global/local asymptotic stability is guaranteed for the optimal equilibrium sets described by the system, the control allocation update-law and the adaptive update-law, if some persistence of exitation condition holds. Simulations of a scaled-model ship, manoeuvred at low-speed, demonstrate the performance of the proposed allocation scheme. $^{*}$
\end{abstract}

Keywords: Control allocation; Adaptive control; Nonlinear systems

\section{Introduction}

Consider the high-level system dynamics

$$
\dot{x}=f(t, x)+g(t, x) \tau
$$

the effector model

$$
\begin{aligned}
\tau & =\Phi(t, x, u, \theta) \\
\Phi(t, x, u, \theta) & :=\Phi_{0}(t, x, u)+\Phi_{\theta_{2}}(t, x, u) \theta_{2}+\Phi_{\theta_{1}}(t, x, u) \theta_{1}
\end{aligned}
$$

and the actuator dynamics

$$
\dot{u}=f_{u 0}\left(t, x, u, u_{c m d}\right)+f_{u \theta}\left(t, x, u, u_{c m d}\right) \theta_{1}
$$

where $t \geq 0, x \in \mathbb{R}^{n}, u \in \mathbb{R}^{r}, \tau \in \mathbb{R}^{d}, \theta:=\left(\theta_{1}^{\mathrm{T}}, \theta_{2}^{\mathrm{T}}\right)^{\mathrm{T}}$, $\theta_{1} \in \mathbb{R}^{m_{1}}, \theta_{2} \in \mathbb{R}^{m_{2}}, u_{c m d} \in \mathbb{R}^{c}$. The constant parameter vectors $\theta_{2}$ and $\theta_{1}$ contains parameters of the

\footnotetext{
* (C) [2007] IEEE. Reprinted, with permission, from Johannes Tjønnås and Tor Arne Johansen, "Optimizing Adaptive Control Allocation With Actuator Dynamics", Proceedings of 46th IEEE Conference on Decision and Control, New Orleans, USA, December 2007.
}

actuator and effector model, that will be viewed as uncertain parameters to be adapted. It is assumed that $x$ and $u$ are measured while $\tau$ is unknown, and $u_{c m d}$ is the input.

This work is motivated by the over-actuated control allocation problem $d \leq r$, where the problem is described by a nonlinear system, divided into a dynamic high-level part (1), a dynamic low-level part (4) and a static part (2). Consider the static optimal control allocation problem:

$$
\min _{u_{d}} J\left(t, x, u_{d}\right) \quad \text { s.t. } \quad \tau_{c}-\Phi\left(t, x, u_{d}+\tilde{u}, \hat{\theta}\right)=0,
$$

where $\hat{\theta}:=\left(\hat{\theta}_{1}^{\mathrm{T}}, \hat{\theta}_{2}^{\mathrm{T}}\right)^{\mathrm{T}}$ is the parameter estimates, $\tilde{u}:=$ $u-u_{d}$ and $u_{d}$ is the actuator reference. The main contribution in this paper is an adaptive allocation algorithm that generates a desired reference $u_{d}$ for the low-level control based on a high level control law $\tau_{c}$, where (5) not necessarily needs to be solved exactly at each time instant.

Optimizing control allocation solutions have been derived for certain classes of over-actuated systems, 
such as aircraft, automotive vehicles and marine vessels, (Enns, 1998; Buffington et al., 1998; Sørdalen, 1997; Bodson, 2002; Härkegård, 2002; Luo et al., 2004, 2005; Poonamallee et al., 2005; Johansen et al., 2004) and (Johansen et al., 2005). The control allocation problem is, in (Enns, 1998; Buffington et al., 1998; Sørdalen, 1997; Johansen et al., 2005; Bodson, 2002) and (Härkegård, 2002), viewed as a static or quasidynamic problem considering non-adaptive linear effector models of the form $\tau=G u$, neglecting the effect of actuator dynamics. In (Luo et al., 2004) and (Luo et al., 2005) a dynamic model predictive approach is considered to solve the allocation problem with linear time-varying dynamics in the actuator model, $T \dot{u}+u=$ $u_{c m d}$. In (Poonamallee et al., 2005) and (Johansen et al., 2004) sequential quadratic programming techniques are used to cope with nonlinearities in the control allocation problem due to singularity avoidance. The main advantage of the control allocation approach is in general the modularity and the ability to handle redundancy and constraints. In the present work we consider dynamic solutions based on the ideas presented in (Johansen, 2004) and (Tjønnås and Johansen, 2005). In (Johansen, 2004) it was shown that it is not necessary to solve the optimization problem (5) exactly at each time instant. Further a control Lyapunov function was used to derive an exponentially convergent update-law for $u$ (related to a gradient or Newton-like optimization) such that the control allocation problem (5) could be solved dynamically. It was also shown that convergence and asymptotic optimality of the system, composed by the dynamic control allocation and a uniform globally exponentially stable trajectory-tracking controller $\tau_{c}$, guarantees uniform boundedness and uniform global exponential convergence to the optimal solution of the system. The advantage of this approach is computational efficiency and simplicity of implementation, since the optimizing control allocation algorithm is implemented as a dynamic nonlinear controller. Solving (5) online at each sampling instant requires a computationally more expensive numerical solution of a nonlinear program in order to guarantee optimality. In (Tjønnås and Johansen, 2005) the results were extended by allowing uncertain parameters, associated with an adaptive law, in the effector model, and by applying set-stability analysis in order to also conclude asymptotic stability of the optimal solution. The results in (Tjønnås and Johansen, 2005) are extended in (Tjønnås and Johansen, 2007) by considering actuator dynamic and relaxing some conditions using the theory in (Tjønnås et al., 2006). In the present paper we extend the result in (Tjønnås and Johansen, 2007 ) by a slightly different parameterization of (2) and (3).
Whenever referring to the notion of set-stability, the set has the property of being nonempty, and we strictly follow the definitions given in (Tjønnås et al., 2006) motivated by (Teel et al., 2002) and (Lin et al., 1996).

\section{Adaptive control allocation with actuator dynamics}

The task of the dynamic control allocation algorithm is to connect the high and low level controls by taking the desired virtual control $\tau_{c}$ as an input and computing the desired actuator reference $u_{d}$ as an output. Based on the minimization problem (5) where $J$ is a cost function that incorporates objectives such as minimum power consumption and actuator constraints (implemented as barrier functions), the Lagrangian function

$$
L\left(t, x, u_{d}, \tilde{u}, \lambda, \hat{\theta}\right):=J\left(t, x, u_{d}\right)+\left(\tau_{c}-\Phi\left(t, x, u_{d}+\tilde{u}, \hat{\theta}\right)\right)^{\mathrm{T}} \lambda
$$

can be introduced. The idea is then to define update laws for the actuator reference $u_{d}$ and the Lagrangian parameter $\lambda$, based on a Lyapunov approach, such that $u_{d}$ and $\lambda$ converges to a set defined by the first order optimal condition for $L$.

Since the parameter vector $\theta$ from the effector and actuator models are unknown, an adaptive update law for $\hat{\theta}$ is defined. The parameter estimates are used in the Lagrangian function (6) and a certainty equivalent adaptive optimal control allocation can be defined. The following observers are used in order to produce estimates of the parameters:

$\dot{\hat{u}}=A_{\hat{u}}(u-\hat{u})+f_{u 0}\left(t, x, u, u_{c m d}\right)+f_{u \theta}\left(t, x, u, u_{c m d}\right) \theta_{1}$
$\dot{\hat{x}}=A_{\hat{x}}(x-\hat{x})+f(t, x)+g(t, x) \Phi(t, x, u, \hat{\theta})$.

where $\left(-A_{\hat{u}}\right)$ and $\left(-A_{\hat{x}}\right)$ are Hurwitz matrices.

In the following, if stating that a function $F$ is uniformly bounded by $y$, this means that there exist a function $G_{F}: \mathbb{R}_{\geq 0} \rightarrow \mathbb{R}_{\geq 0}$ such that $|F(t, y, z)|<$ $G_{f}(|y|)$ for all $y, z$ and $t$.

\section{Assumpiton 1 (Plant)}

a) The states from (1) and (4) are known for all $t$.

b) The function $f$ is uniformly locally Lipschitz in $x$ and uniformly bounded by $x$. The function $g$ is uniformly bounded and it's partial derivatives are bounded by $x$.

c) The function $\Phi$ is twice differentiable and uniformly bounded by $x$ and $u$. Moreover it's partial derivatives are uniformly bounded by $x$. 
d) There exists constants $\varrho_{2}>\varrho_{1}>0$, such that $\forall t, x$, $u$ and $\theta$

$$
\varrho_{1} I \leq \frac{\partial \Phi}{\partial u}(t, x, u, \theta)\left(\frac{\partial \Phi}{\partial u}(t, x, u, \theta)\right)^{T} \leq \varrho_{2} I .
$$

Assumpiton 2 (High and Low level Controller Algorithms)

a) There exists a high level control $\tau_{c}:=k(t, x)$, that render the equilibrium of (1) UGAS for $\tau=\tau_{c}$. The function $k$ is uniformly bounded by $x$ and differentiable. It's partial derivatives are uniformly bounded by $x$.

b) There exists a low-level control

$u_{c m d}:=k_{u}\left(t, x, u, u_{d}, \dot{u}_{d}, \hat{\theta}_{1}\right)$ that makes the equilibrium of

$$
\dot{\tilde{u}}=f_{\tilde{u}}\left(t, x, \tilde{u}, u_{d}, \hat{\theta}_{1}, \theta_{1}\right)
$$

$U G A S$ if $\hat{\theta}_{1}=\theta_{1}$ and $x, u_{d}, \dot{u}_{d}$ exist for all $t>0$, where

$$
\begin{aligned}
& f_{\tilde{u}}\left(t, x, \tilde{u}, u_{d}, \hat{\theta}_{1}, \theta_{1}\right):= \\
& +f_{u 0}\left(t, x, u, k_{u}\left(t, x, u, u_{d}, \dot{u}_{d}(t), \hat{\theta}_{1}\right)\right) \\
& +f_{u \theta}\left(t, x, u, k_{u}\left(t, x, u, u_{d}, \dot{u}_{d}(t), \hat{\theta}_{1}\right)\right) \theta_{1} \\
& -k_{u}\left(t, x, u, u_{d}, \dot{u}_{d}(t), \hat{\theta}_{1}\right) .
\end{aligned}
$$

Remark 1 From assumption 2a) there exist a Lyapunov function $V_{x}: \mathbb{R}_{\geq 0} \times \mathbb{R}^{n} \mapsto \mathbb{R}_{\geq 0}$ and $\mathcal{K}_{\infty}$ functions $\alpha_{x 1}, \alpha_{x 2}, \alpha_{x 3}$ and $\alpha_{x 4}$ such that

$$
\begin{aligned}
\alpha_{x 1}(|x|) \leq V_{x}(t, x) & \leq \alpha_{x 2}(|x|) \\
\frac{\partial V_{x}}{\partial t}+\frac{\partial V_{x}}{\partial x}(f(t, x)+g(t, x) k(t, x)) & \leq \alpha_{x 3}(|x|) \\
\left|\frac{\partial V_{x}}{\partial x}\right| & \leq \alpha_{x 4}(|x|) .
\end{aligned}
$$

We will not discuss the details in these assumptions, but they are sufficient in order to guarantee existence of solutions and validity of the update-laws that we propose in this paper, see (Tjønnås and Johansen, 2005). The main problem formulation is given by:

Problem: Define update-laws (14)-(16) for $u_{d}, \lambda$ and $\hat{\theta}$, such that the stability of the closed loop:

$$
\begin{aligned}
\dot{x} & =f(t, x)+g(t, x) k(t, x) \\
& +g(t, x)(\Phi(t, x, u, \theta)-k(t, x)) \\
\dot{\tilde{u}} & =f_{\tilde{u}}\left(t, x, \tilde{u}, u_{d}, \hat{\theta}_{1}, \theta_{1}\right) \\
\dot{u}_{d} & :=f_{d}\left(t, x, \tilde{u}, u_{d}, \hat{\theta}\right) \\
\dot{\lambda} & :=f_{\lambda}\left(t, x, \tilde{u}, u_{d}, \hat{\theta}\right) \\
\dot{\tilde{\theta}} & :=-f_{\hat{\theta}}\left(t, x, \tilde{u}, u_{d}, \hat{\theta}\right) \\
\dot{\eta}_{u} & =-A_{\hat{u}} \eta_{u}+\bar{f}_{u \theta}\left(t, x, u_{d}, \tilde{u}, \hat{\theta}\right) \tilde{\theta}_{1} \\
\dot{\eta}_{x} & =-A_{\hat{x}} \eta_{x}+\Phi_{\theta_{2}}(t, x, u) \tilde{\theta}_{2}+\Phi_{\theta_{1}}(t, x, u) \tilde{\theta}_{1}
\end{aligned}
$$

where $\bar{f}_{u \theta}\left(t, x, u_{d}, \tilde{u}, \hat{\theta}\right):=f_{u \theta}\left(t, x, u, k_{u}\left(t, x, u, u_{d}\right.\right.$, $\left.\left.f_{d}\left(t, x, \tilde{u}, u_{d}, \hat{\theta}\right), \hat{\theta}_{1}\right)\right), \tilde{\theta}=\theta-\hat{\theta}, \eta_{u}:=u-\hat{u}, \eta_{x}:=x-\hat{x}$, is conserved and $u_{d}(t)$ converges to an optimal solution with respect to the minimization problem (5).

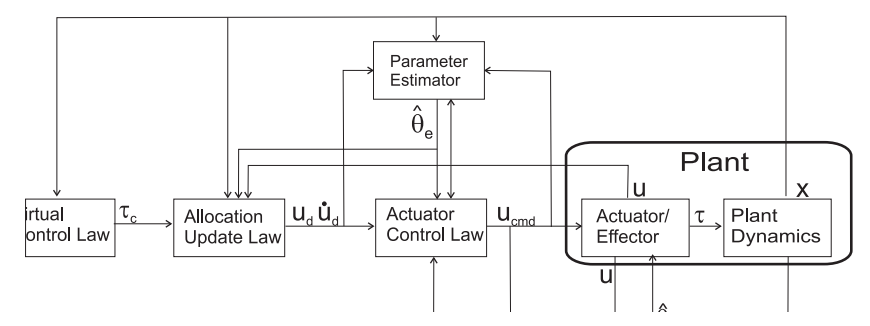

Figure 1: The closed loop diagram of the certainty equivalent control allocation algorithm

Let (12) define the sub-system $\Sigma_{1}$ and (13)-(18) define the sub-system $\Sigma_{2}$, then $\Sigma_{1}$ and $\Sigma_{2}$ form a cascade as long as $x(t)$ exists for all $t>0$, and is viewed as a time-varying input to $\Sigma_{2}$. For the system $\Sigma_{2}$ we will consider stability with respect to the set

$$
\mathcal{O}_{u_{d} \lambda \tilde{\theta}}(t, x):=\left\{z_{u_{d} \lambda \tilde{\theta}} \in \mathbb{R}^{n_{u_{d} \lambda \tilde{\theta}}} \mid f_{\mathcal{O}_{u \lambda \tilde{\theta}}}\left(t, x, z_{u_{d} \lambda \tilde{\theta}}\right)=0\right\}
$$

where $n_{u_{d} \lambda \tilde{\theta}}:=3 r+d+n+m$,

$z_{u_{d} \lambda \tilde{\theta}}:=\left(u_{d}^{\mathrm{T}}, \lambda^{\mathrm{T}}, \tilde{u}^{\mathrm{T}}, \eta_{u}^{\mathrm{T}}, \eta_{x}^{\mathrm{T}}, \tilde{\theta}^{\mathrm{T}}\right)^{\mathrm{T}}$ and $f_{\mathcal{O}_{u \lambda \tilde{\theta}}}\left(t, x, z_{u_{d} \lambda \tilde{\theta}}\right):=$ $\left(\left(\frac{\partial L}{\partial u}\right)^{\mathrm{T}},\left(\frac{\partial L}{\partial \lambda}\right)^{\mathrm{T}}, \tilde{u}^{\mathrm{T}}, \eta_{u}^{\mathrm{T}}, \eta_{x}^{\mathrm{T}}, \tilde{\theta}^{\mathrm{T}}\right)$. In order to relate the notion of optimal control allocation to the set $\mathcal{O}_{u_{d} \lambda \tilde{\theta}}(t, x)$, we introduce the sufficient conditions for the set

$$
\begin{aligned}
& \mathcal{O}_{u_{d} \lambda}(t, x, \tilde{u}, \hat{\theta}):= \\
& \left\{\left(u_{d}^{\mathrm{T}}, \lambda^{\mathrm{T}}\right)^{\mathrm{T}} \in \mathbb{R}^{r+d} \mid\left(\left(\frac{\partial L}{\partial u_{d}}\right)^{\mathrm{T}},\left(\frac{\partial L}{\partial \lambda}\right)^{\mathrm{T}}\right)^{\mathrm{T}}=0\right\}
\end{aligned}
$$

to be the optimal solution of problem (5), by the following assumption.

\section{Assumpiton 3 (Optimal Control Allocation)}

a) The cost function $J: \mathbb{R}_{\geq t_{0}} \times \mathbb{R}^{n \times r} \rightarrow \mathbb{R}$ is twice differentiable and $J\left(t, x, u_{d}\right) \rightarrow \infty$ as $\left|u_{d}\right| \rightarrow \infty$. Furthermore $\frac{\partial J}{\partial u_{d}}, \frac{\partial^{2} J}{\partial t \partial u_{d}}$ and $\frac{\partial^{2} J}{\partial x \partial u_{d}}$ are uniformly bounded by $x$ and $u_{d}$.

b) There exists constants $k_{2}>k_{1}>0$, such that $\forall t$, $x, \hat{\theta}, \tilde{u}$ and $\left(u_{d}^{T}, \lambda^{T}\right)^{T} \notin \mathcal{O}_{u_{d} \lambda}(t, x, \tilde{u}, \hat{\theta})$

$$
k_{1} I \leq \frac{\partial^{2} L}{\partial u_{d}^{2}}\left(t, x, u_{d}, \tilde{u}, \lambda, \hat{\theta}\right) \leq k_{2} I
$$


If $\left(u_{d}^{T}, \lambda^{T}\right)^{T} \in \mathcal{O}_{u_{d} \lambda}(t, x, \tilde{u}, \hat{\theta})$ the lower bound is replaced by $\frac{\partial^{2} L}{\partial u_{d}^{2}} \geq 0$

Lemma 1 By Assumption 1 there exists continuous functions $\varsigma_{x}, \varsigma_{x u}, \varsigma_{u}: \mathbb{R}_{\geq 0} \rightarrow \mathbb{R}_{\geq 0}$, such that

$$
\begin{gathered}
\left|\Phi_{\theta 1}\left(t, x, \tilde{u}+u_{d}\right)\right|+\left|\Phi_{\theta 2}\left(t, x, \tilde{u}+u_{d}\right)\right| \\
\leq \varsigma_{x}(|x|) \varsigma_{x x}(|x|) \varsigma_{x u}(|\tilde{u}|)+\varsigma_{x}(|x|) \varsigma_{u}\left(\left|z_{u_{d} \lambda \tilde{\theta}}\right|_{\mathcal{O}_{u_{d} \lambda \tilde{u} \tilde{\theta}}}\right) .
\end{gathered}
$$

\section{Assumpiton 2 (continued)}

c) There exists a $\mathcal{K}_{\infty}$ function $\alpha_{k}: \mathbb{R}_{\geq 0} \rightarrow \mathbb{R}_{\geq 0}$, such that

$$
\alpha_{k}^{-1}(|x|) \alpha_{x 3}(|x|) \geq \alpha_{x 4}(|x|) \bar{\varsigma}_{x}(|x|),
$$

$$
\text { where } \bar{\varsigma}_{x}(|x|):=\max \left(1, \varsigma_{x}(|x|), \varsigma_{x}(|x|) \varsigma_{x x}(|x|)\right) \text {. }
$$

We approach the problem formulation by i) defining a Lyapunov like function, $V_{u_{d} \lambda \tilde{u} \eta \tilde{\theta}}$, for the system $\Sigma_{2}$ and defining explicit update-laws for $u_{d}, \lambda$ and $\tilde{\theta}$ such that $\dot{V}_{u_{d} \lambda \tilde{u} \eta \tilde{\theta}} \leq 0$. ii) Furthermore, boundedness of the closed-loop system, $\Sigma_{1}$ and $\Sigma_{2}$ can be proved, and the cascade lemma from (Tjønnås et al., 2006) can be applied to prove convergence and stability.

Consider the Lyapunov function candidate

$V_{u_{d} \lambda \tilde{u} \eta \tilde{\theta}}\left(t, x, u_{d}, \lambda, \tilde{u}, \eta\right):=V_{\tilde{u}}(t, \tilde{u})+\frac{1}{2} \eta_{u}^{\mathrm{T}} \Gamma_{\eta} \eta_{u}+\frac{1}{2} \eta_{x}^{\mathrm{T}} \Gamma_{\tilde{x}} \eta_{x}$

$+\frac{1}{2}\left(\frac{\partial L^{\mathrm{T}}}{\partial u_{d}} \frac{\partial L}{\partial u_{d}}+\frac{\partial L^{\mathrm{T}}}{\partial \lambda} \frac{\partial L}{\partial \lambda}\right)+\frac{1}{2} \tilde{\theta}_{1}^{\mathrm{T}} \Gamma_{\theta_{1}} \tilde{\theta}_{1}+\frac{1}{2} \tilde{\theta}_{2}^{\mathrm{T}} \Gamma_{\theta_{2}} \tilde{\theta}_{2}$

and the algorithm:

$$
\begin{gathered}
\left(\begin{array}{c}
\dot{u}_{d} \\
\dot{\lambda}
\end{array}\right)=-\Gamma \mathbb{H}\left(\begin{array}{c}
\frac{\partial L_{\hat{\theta}}}{\partial u_{d}} \\
\frac{\partial L_{\hat{\theta}}}{\partial \lambda}
\end{array}\right)-u_{f f} \\
\dot{\hat{\theta}}_{1}^{\mathrm{T}}=\left(\frac{\partial V_{\tilde{u}}}{\partial \tilde{u}}+\eta_{u}^{\mathrm{T}} \Gamma_{\eta}\right) f_{u \theta}\left(t, x, u_{d}+\tilde{u}, u_{c m d}\right) \Gamma_{\theta_{1}}^{-1} \\
+\left(\tilde{x}^{\mathrm{T}} \Gamma_{\tilde{x}}+\frac{\partial L^{\mathrm{T}}}{\partial u_{d}} \frac{\partial^{2} L}{\partial \tilde{u} \partial u_{d}}+\frac{\partial L^{\mathrm{T}}}{\partial \lambda} \frac{\partial^{2} L}{\partial \tilde{u} \partial \lambda}\right) f_{u \theta}\left(t, x, u, u_{c m d}\right) \Gamma_{\theta_{1}}^{-1} \\
+\left(\frac{\partial L^{\mathrm{T}} \partial^{2} L}{\partial u_{d}} \frac{\partial L^{\mathrm{T}}}{\partial x \partial u_{d}}+\frac{\partial^{2} L}{\partial \lambda} \partial x \partial \lambda\right) g(t, x) \Phi_{\theta_{1}}\left(t, x, u_{d}+\tilde{u}\right) \Gamma_{\theta_{1}}^{-1}
\end{gathered}
$$

$$
\begin{aligned}
& \dot{\hat{\theta}}_{2}^{\mathrm{T}}=\eta_{x}^{\mathrm{T}} \Gamma_{\tilde{x}} g(t, x) \Phi_{\theta_{2}}(t, x, u) \Gamma_{\theta_{2}}^{-1} \\
& +\left(\frac{\partial L^{\mathrm{T}}}{\partial u_{d}} \frac{\partial^{2} L}{\partial x \partial u_{d}}+\frac{\partial L^{\mathrm{T}}}{\partial \lambda} \frac{\partial^{2} L}{\partial x \partial \lambda}\right) g(t, x) \Phi_{\theta_{2}}(t, x, u) \Gamma_{\theta_{2}}^{-1}
\end{aligned}
$$

where $\mathbb{H}:=\left(\begin{array}{cc}\frac{\partial^{2} L}{\partial u_{d}^{2}} & \frac{\partial^{2} L}{\partial \lambda \partial u_{d}} \\ \frac{\partial^{2} L}{\partial u_{d} \partial \lambda} & 0\end{array}\right), \Gamma$ is a possibly timevarying symmetric positive definite weighting matrix and $u_{f f}$ is a feed-forward like term:

$$
\begin{aligned}
& u_{f f}:=\mathbb{H}^{-1}\left(\begin{array}{c}
\frac{\partial^{2} L}{\partial t \partial u_{d}} \\
\frac{\partial^{2} L}{\partial t \partial \lambda}
\end{array}\right)+\mathbb{H}^{-1}\left(\begin{array}{c}
\frac{\partial^{2} L}{\partial x \partial u_{d}} \\
\frac{\partial^{2} L}{\partial x \partial \lambda}
\end{array}\right) f(t, x) \\
& +\mathbb{H}^{-1}\left(\begin{array}{c}
\frac{\partial^{2} L}{\partial x \partial u_{d}} \\
\frac{\partial^{2} L}{\partial x \partial \lambda}
\end{array}\right) g(t, x)\left(k(t, x)-\Phi\left(t, x, u_{d}+\tilde{u}, \hat{\theta}\right)\right) \\
& +\mathbb{H}^{-1}\left(\begin{array}{c}
\frac{\partial^{2} L}{\partial \tilde{u} \partial u_{d}} \\
\frac{\partial^{2} L}{\partial \tilde{u} \partial \lambda}
\end{array}\right) f_{\tilde{u}}\left(t, x, \tilde{u}, u_{d}, u_{c m d}, \hat{\theta}\right)+\mathbb{H}^{-1}\left(\begin{array}{c}
\frac{\partial^{2} L}{\partial \hat{\theta} \partial u_{d}} \\
\frac{\partial^{2} L}{\partial \hat{\theta} \partial \lambda}
\end{array}\right) \dot{\hat{\theta}}
\end{aligned}
$$

if $\operatorname{det}(\mathbb{H}) \neq 0$ and $u_{f f}:=0$ if $\operatorname{det}(\mathbb{H})=0$, then the time derivative of $V_{u_{d} \lambda \tilde{u} \eta \tilde{\theta}}$ along the trajectories of $\Sigma_{1}$ and $\Sigma_{2}$ is given by:

$$
\begin{aligned}
& \dot{V}_{u_{d} \lambda \tilde{u} \eta \tilde{\theta}}=-\eta^{\mathrm{T}} \Gamma_{\eta} A \eta-\alpha_{\tilde{u} 3}(|\tilde{u}|)-\tilde{x}^{\mathrm{T}} \Gamma_{\tilde{x}} A_{\tilde{x}} \tilde{x} \\
& -\left(\frac{\partial L^{\mathrm{T}}}{\partial u_{d}}, \frac{\partial L^{\mathrm{T}}}{\partial \lambda}\right) \mathbb{H} \Gamma \mathbb{H}\left(\frac{\partial L^{\mathrm{T}}}{\partial u_{d}}, \frac{\partial L^{\mathrm{T}}}{\partial \lambda}\right)^{\mathrm{T}} .
\end{aligned}
$$

Proposition 1 If the assumptions 1, 2 and 3 are satisfied, then the solution of the closed-loop (12)-(18) is bounded with respect to a set $\mathcal{O}_{x u_{d} \lambda \tilde{\theta}}(t):=\mathcal{O}_{u_{d} \lambda \tilde{\theta}}(t, 0) \times$ $\left\{x \in \mathbb{R}_{\geq t_{0}} \mid x=0\right\}$. Furthermore the set $\mathcal{O}_{x u_{d} \lambda \tilde{\theta}}$ is UGS with respect to the system defined by (12)-(18). If in addition $f_{p}(t):=f_{u \theta}\left(t, x(t), u(t), u_{c m d}(t)\right)$ and $\Phi_{g}(t):=$ $g(t, x(t)) \Phi_{\theta_{2}}(t, x(t), u(t))$ are Persistently Exited $(P E)$, i.e. there exist constants $T$ and $\gamma>0$, such that

$$
\int_{t}^{t+T} F(\tau)^{T} F(\tau) d \tau \geq \gamma I, \quad \forall t>t_{0},
$$

is satisfied for $F(\tau)=f_{p}(t)$ and $F(\tau)=\Phi_{g}(t)$, then the set $\mathcal{O}_{x u_{d} \lambda \tilde{\theta}}$ is UGAS with respect to the system (12)(18).

The proof of Proposition 1 involves similar steps as in the proof of the main result in (Tjønnås and Johansen, 2007) and is therefore omitted here.

Proposition 1 implies that the time-varying first order optimal set $\mathcal{O}_{x u \lambda \tilde{\theta}}(t)$ is uniformly stable, and in addition uniformly attractive if a PE assumption is satisfied. Thus adaptive optimal control allocation is achieved asymptotically for the closed loop under the PE condition.

Corollary 1 If for $\mathbb{U} \subset \mathbb{R}^{r}$ there exist constant $c_{x}>0$ such that for $|x| \leq c_{x}$ the domain $\mathbb{U}_{z} \subset \mathbb{R}^{n} \times \mathbb{U} \times$ $\mathbb{R}^{2 r+d+n+m}$ contain $\mathcal{O}_{x u_{d} \lambda \tilde{\theta}}$, then if the Assumptions 1 3 are satisfied, the set $\mathcal{O}_{x u_{d} \lambda \tilde{\theta}}$ is US with respect to the system (12)-(18). If in addition $f_{p}(t)$ and $\Phi_{g}(t)$ are $P E, \mathcal{O}_{x u \lambda \tilde{\theta}}$ is UAS with respect to the system (12)-(18). 


\section{Example}

In this section, simulation results of an over-actuated scaled-model ship, manoeuvred at low-speed, is presented. The scale model-ship is moved while experiencing disturbances caused by wind and current, and propellers trust losses. The propeller losses can be due to: Axial Water Inflow, Cross Coupling Drag, ThrusterHull and Thruster-Thruster Interaction (see (Sørensen et al., 1997) and (Fossen and Blanke, 2000) for details). But in this example we limit our study to thruster loss caused by Thruster-Hull interaction. A 3DOF horizontal plane model described by:

$$
\begin{aligned}
\dot{\eta}_{e} & =R\left(\psi_{p}\right) \nu \\
\dot{\nu} & =-M^{-1} D \nu+M^{-1} \tau \\
\tau & =\Phi(\nu, u, \theta),
\end{aligned}
$$

is considered, where $\eta_{e}:=\left(x_{e}, y_{e}, \psi_{e}\right)^{\mathrm{T}}:=\left(x_{p}-x_{d}, y_{p}-\right.$ $\left.y_{d}, \psi_{p}-\psi_{d}\right)^{\mathrm{T}}$ is the north and east positions and compass heading deviations. Subscript $p$ and $d$ denotes the actual and desired states. $\nu:=\left(v_{x}, v_{y}, r\right)^{\mathrm{T}}$ is the body-fixed velocities, surge, sway and yaw, $\tau$ is the generalized force vector and $R\left(\psi_{p}\right)$ is the rotation matrix function between the body fixed and the earth fixed coordinate frame. The example we present here is based on (Lindegaard and Fossen, 2003), and is also studied in (Johansen, 2004), (Tjønnås and Johansen, 2005) and (Tjønnås and Johansen, 2007). In the considered model there are five force producing devices; the two main propellers aft of the hull, in conjunction with two rudders, and one tunnel thruster going through the hull of the vessel. $\omega_{i}$ denotes the propeller angular velocity and $\delta_{i}$ denotes the rudder deflection. $i=1,2$ denotes the aft actuators, while $i=3$ denotes the tunnel thruster. Equation (27) can be rewritten in the form of (1) and (2) by:

$$
\begin{aligned}
& x:=\left(\eta_{e}, \nu\right)^{\mathrm{T}}, \theta_{1}:=\left(\theta_{11}, \theta_{12}, \theta_{13}\right)^{\mathrm{T}}, \theta_{2}:=\left(\theta_{21}, \theta_{22}, \theta_{23}\right)^{\mathrm{T}} \\
& \tau:=\left(\tau_{1}, \tau_{2}, \tau_{3}\right)^{\mathrm{T}}, u:=\left(\omega_{1}, \omega_{2}, \omega_{3}, \delta_{1}, \delta_{2}\right)^{\mathrm{T}}, \\
& f:=\left(\begin{array}{c}
R\left(\psi_{e}+\psi_{d}\right) \nu \\
-M^{-1} D \nu
\end{array}\right), g:=\left(\begin{array}{c}
0 \\
M^{-1}
\end{array}\right) \text {, } \\
& \Phi(\nu, u, \theta):=G_{u}(u)\left(\begin{array}{c}
T_{1}\left(v_{x}, \omega_{1}, \theta_{11}\right) \\
T_{2}\left(v_{x}, \omega_{2}, \theta_{12}\right) \\
T_{3}\left(v_{x}, v_{y}, \omega_{3}, \theta_{13}\right)
\end{array}\right)+R\left(\psi_{p}\right) \theta_{2} \\
& G_{u}(u):=\left(\begin{array}{ccc}
\left(1-D_{1}\right) & \left(1-D_{2}\right) & 0 \\
L_{1} & L_{2} & 1 \\
\Phi_{31} & \Phi_{32} & l_{3, x}
\end{array}\right) \\
& \Phi_{31}(u):=-l_{1, y}\left(1-D_{1}(u)+l_{1, x} L_{1}(u)\right), \\
& \Phi_{32}(u):=-l_{2, y}\left(1-D_{2}(u)+l_{2, x} L_{2}(u)\right) \text {. }
\end{aligned}
$$

The thruster forces are given by:

$$
\begin{aligned}
& T_{i}\left(v_{x}, \omega_{i}, \theta_{1 i}\right):=T_{n i}\left(\omega_{i}\right)-\phi_{i}\left(\omega_{i}, v_{x}\right) \theta_{1 i} \\
& T_{n i}\left(\omega_{i}\right):=\left\{\begin{array}{cc}
k_{T p_{i}} \omega_{i}^{2} & \omega_{i} \geq 0 \\
k_{T n_{i}}\left|\omega_{i}\right| \omega_{i} & \omega_{i}<0
\end{array},\right. \\
& \phi_{1}\left(\omega_{1}, v_{x}\right):=\omega_{1} v_{x}, \quad \phi_{2}\left(\omega_{2}, v_{x}\right):=\omega_{2} v_{x} \\
& \phi_{3}\left(\omega_{3}\right):=\sqrt{\left.v_{x}^{2}+v_{y}^{2}\right)}\left|\omega_{3}\right| \omega_{3}, \quad \theta_{13}:=k_{T \theta_{3}} \\
& \theta_{11}:=\left\{\begin{array}{cc}
k_{T \theta_{1}}(1-w) & v_{x} \geq 0 \\
k_{T \theta_{1}} & v_{x}<0
\end{array},\right. \\
& \theta_{12}:=\left\{\begin{array}{cc}
k_{T \theta_{2}}(1-w) & v_{x} \geq 0 \\
k_{T \theta_{2}} & v_{x}<0
\end{array},\right.
\end{aligned}
$$

where $0<w<1$ is the wake fraction number, $\phi_{i}\left(\omega_{i}, v_{x}\right) \theta_{1 i}$ is the thrust loss due to changes in the advance speed, $v_{a}=(1-w) v_{x}$, and the unknown parameters $\theta_{1 i}$ represents the thruster loss factors dependent on whether the hull invokes on the inflow of the propeller or not. The rudder lift and drag forces are projected through:

$$
\begin{aligned}
& L_{i}(u):= \begin{cases}\left(1+k_{L n_{i}} \omega_{i}\right)\left(k_{L \delta 1_{i}}+k_{L \delta 2_{i}}\left|\delta_{i}\right|\right) \delta_{i} & , \omega_{i} \geq 0 \\
0 & , \omega_{i}<0\end{cases} \\
& D_{i}(u):= \begin{cases}\left(1+k_{D n_{i}} \omega_{i}\right)\left(k_{D \delta 1_{i}}\left|\delta_{i}\right|+k_{D \delta 2_{i}} \delta_{i}^{2}\right) & , \omega_{i} \geq 0 \\
0 & , \omega_{i}<0\end{cases}
\end{aligned}
$$

Further more it is clear from (28) that $\Phi(\nu, u, \theta)=$ $G_{u}(u) Q(u)+G_{u}(u) \phi\left(\omega, v_{x}\right) \theta_{1}+R\left(\psi_{e}\right) \theta_{2}$, where $\phi\left(\omega, v_{x}\right):=$ $\operatorname{diag}\left(\phi_{1}, \phi_{2}, \phi_{3}\right), Q(u)$ represents the nominal propeller thrust and $\theta_{2}$ represents unknown external disturbances, such as ocean current, that are constant in the earth fixed coordinate frame.

The actuator error dynamic for each propeller is based on the propeller model presented in (Pivano et al., 2007) and given by

$$
\begin{aligned}
J_{m i} \dot{\tilde{\omega}}_{i} & =-k_{f i}\left(\tilde{\omega}_{i}+\omega_{d i}\right)-\frac{T_{n i}}{a_{T}}\left(\tilde{\omega}_{i}+\omega_{d i}\right) \\
& +\frac{\phi_{i}\left(\omega_{i}, v_{x}\right) \theta_{1 i}}{a_{T}}+u_{c m d i}-J_{m i} \dot{\omega}_{d i}
\end{aligned}
$$

where $\tilde{\omega}_{i}:=\left(\omega_{i}-\omega_{i d}\right), J_{m}$ is the shaft moment of inertia, $k_{f}$ is a positive coefficient related to the viscous friction, $a_{T}$ is a positive model constant (Pivano et al., 2006) and $u_{c m d}$ is the commanded motor torque. By the quadratic Lyapunov function $\frac{\tilde{\omega}_{i}^{2}}{2}$ it is easy to see that the control law

$$
\begin{aligned}
u_{c m d i}:= & -K_{\omega p}\left(\tilde{\omega}_{i}\right)-\frac{\phi_{i}\left(\omega_{i}, v_{x}\right) \hat{\theta}_{1 i}}{a_{T}}+J_{m i} \dot{\omega}_{d i} \\
& +\frac{T_{n i}\left(\omega_{d i}\right)}{a_{T}}+k_{f i} \omega_{d i} .
\end{aligned}
$$

makes the origin of (29) UGES when $\hat{\theta}_{1 i}=\theta_{1 i}$. The rudder model is linearly time-variant and the error dynamic is given by:

$$
m_{i} \dot{\tilde{\delta}}=a_{i}(t)\left(\tilde{\delta}+\delta_{d i}\right)+b_{i} u_{c m d \delta i}-m_{i} \dot{\delta}_{d i}
$$


where $\tilde{\delta}:=\delta_{i}-\delta_{d i}, a_{i}, b_{i}$ are a known scalar parameter bounded away from zero, and the controller

$$
b_{i} u_{c m d \delta i}:=-K_{\delta} \tilde{\delta}-a_{i}(t)\left(\tilde{\delta}+\delta_{d i}\right)+m_{i} \dot{\delta}_{d i}
$$

makes the origin of (31) UGES. The parameters for the actuator model and controllers are: $a_{T}=1, J_{m i}=$ $10^{-2}, k_{f i}=10^{-4}, a_{i}=-10^{-4}, b_{i}=10^{-5}, m_{i}=10^{-2}$, $K_{\omega p}=5 \cdot 10^{-3}$ and $K_{\delta}=10^{-3}$

A virtual controller $\tau_{c}$ that stabilizes the system (27) uniformly, globally and exponentially, for some physically limited yaw rate, is proposed in (Lindegaard and Fossen, 2003) and given by

$$
\tau_{c}:=-K_{i} R^{T}\left(\psi_{p}\right) \xi-K_{p} R^{T}\left(\psi_{p}\right) \eta_{e}-K_{d} \nu,
$$

where (27) is augmented with the integral action described by, $\dot{\xi}=\eta_{e}$. Thus Assumption 2 concerning high- and low- level control is satisfied. The cost function designed for the optimization problem, (5), is:

$$
\begin{aligned}
& J(u):=\sum_{i=1}^{3} k_{i}\left|\omega_{i}\right| \omega_{i}^{2}+k_{i 2} \omega_{i}^{2}+\sum_{i=1}^{2} q_{i} \delta_{i}^{2}-\varsigma \sum_{i=1}^{3} \lg \left(-\omega_{i}+18\right) \\
& -\sum_{i=1}^{3} \lg \left(\omega_{i}+18\right)-\varsigma \sum_{i=1}^{2} \lg \left(-\delta_{i}+35\right)-\sum_{i=1}^{2} \lg \left(\delta_{i}+35\right), \\
& \varsigma=0.05, \quad k_{1}=k_{2}=0.01, \quad k_{3}=0.02, \quad k_{i 2}=10^{-3}, \\
& q_{1}=q_{2}=2500 .
\end{aligned}
$$

By investigating the given specifications of the system we can see that the Assumption 3 is also satisfied locally, since $u$ is bounded. The gain matrices are chosen as follows: $K_{p}:=M \cdot \operatorname{diag}(3.13,3.13,12.5) 10^{-2}, K_{d}:=$ $M \cdot \operatorname{diag}(3.75,3.75,7.5) 10^{-1}, K_{I}:=M \cdot \operatorname{diag}(0.2,0.2,4) 10^{-}$ $A_{\hat{x}}:=10, \Gamma_{\tilde{x}}:=I_{9 \times 9}, \Gamma_{\theta_{2}}^{-1}:=10^{-4} \operatorname{diag}(1,1,10), A_{\hat{u}}:=$ $2 I_{5 \times 5}, \Gamma_{\theta_{1}}:=10^{-3}, \Gamma_{\eta}:=\operatorname{diag}\left(10^{3}, 10^{3}, 3\right)$ and $\Gamma:=$ $\left(\mathbb{H}_{\hat{\theta}}^{\mathrm{T}} W \mathbb{H}_{\hat{\theta}}+\varepsilon I\right)^{-1}$ where $W:=\operatorname{diag}(1,1,1,1,1,0.9,0.9,0.7)$ and $\varepsilon:=10^{-9}$.

The thruster loss vector $\theta_{1}$ and $\hat{\theta}_{1}$ are given in Figure $6, \theta_{2}:=(0.05,0.08,0.02)$ and $\hat{\theta}_{2}$ are given in Figure 7.

The simulation results are presented in the Figures 2-8. The control objective is satisfied and the commanded virtual controls are tracked by the forces generated by the adaptive control allocation law: see Figure 5. Note that there are some deviations since $\omega$ saturates from $0-230 s$ and since the loss parameter has changed at ca. 420s. Also note that the parameter estimates $\hat{\theta}_{1}$ only converge to the true values when the ship is moving and the thrust loss is not zero. The simulations are carried out in a discrete MATLAB environment with a sampling rate of $20 \mathrm{~Hz}$

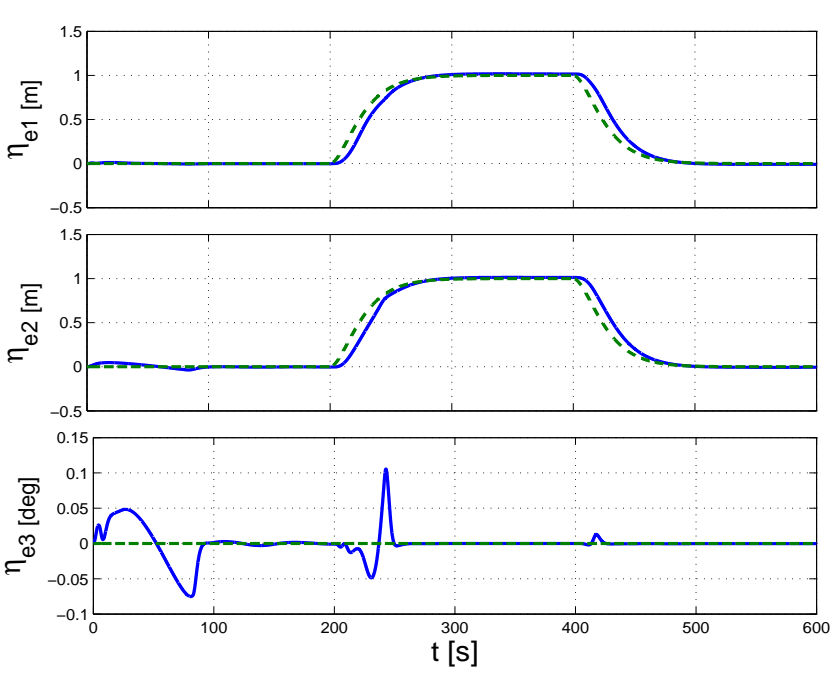

Figure 2: Desired (dashed) and actual ship positions (solid).

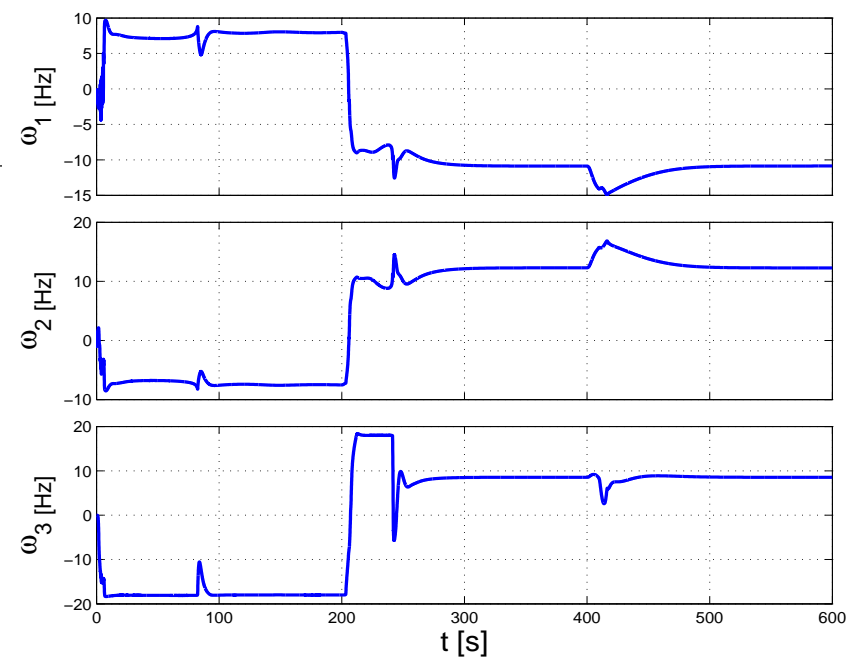

Figure 3: Actual propeller velocities 

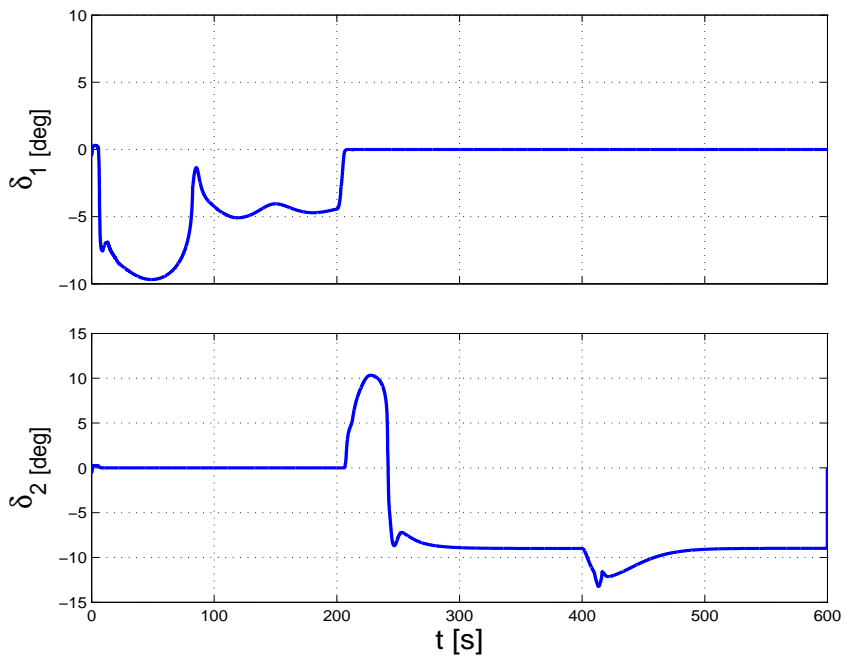

Figure 4: Actual rudder deflection
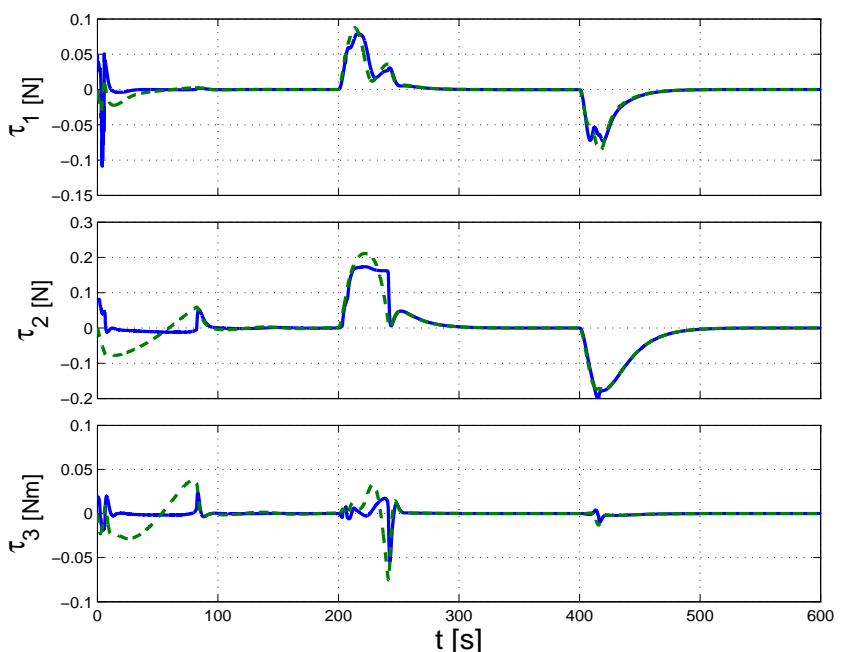

Figure 5: The virtual control (dashed) and actual (solid) forces generated by the actuators

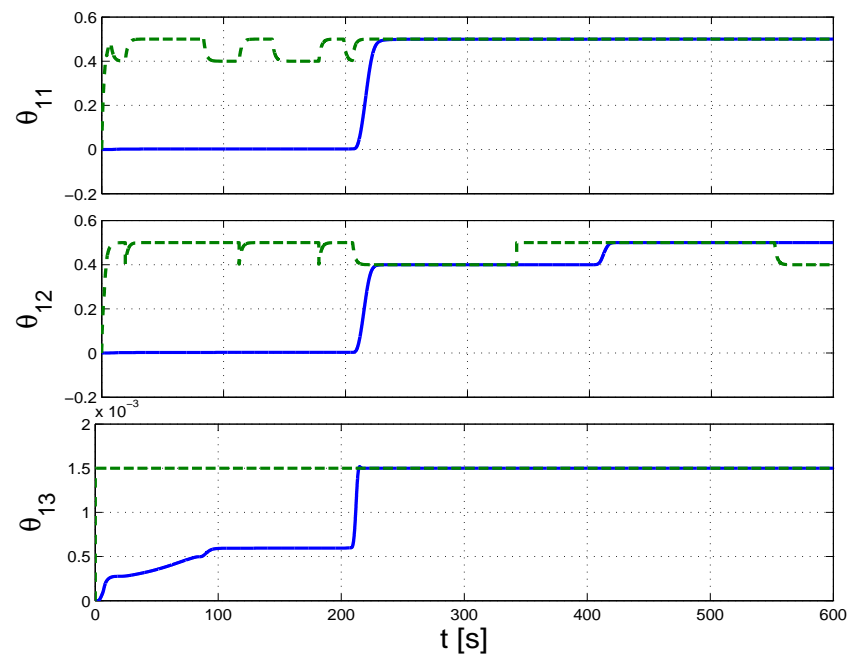

Figure 6: Actual (dashed) and estimated (solid) loss parameters

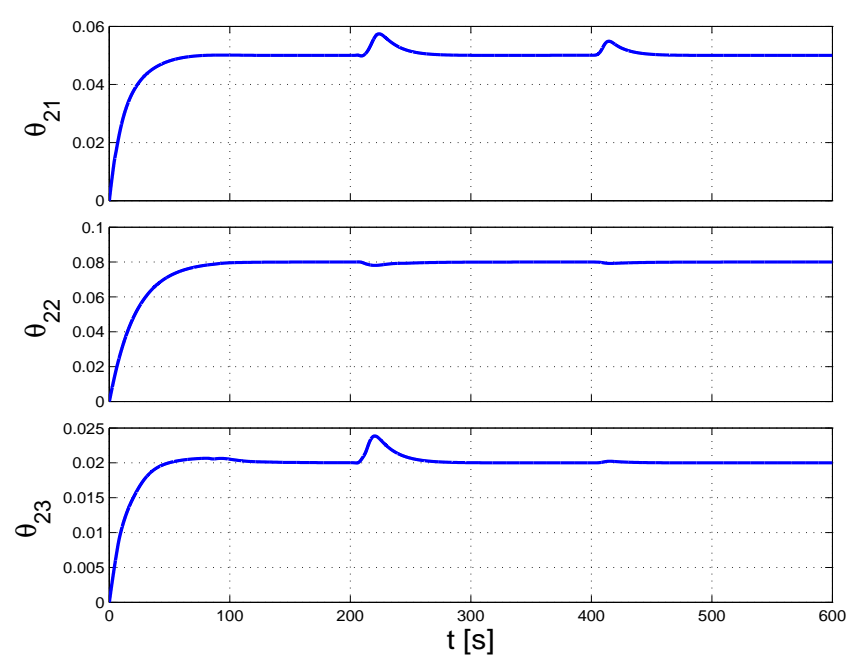

Figure 7: Effector model parameter estimates 


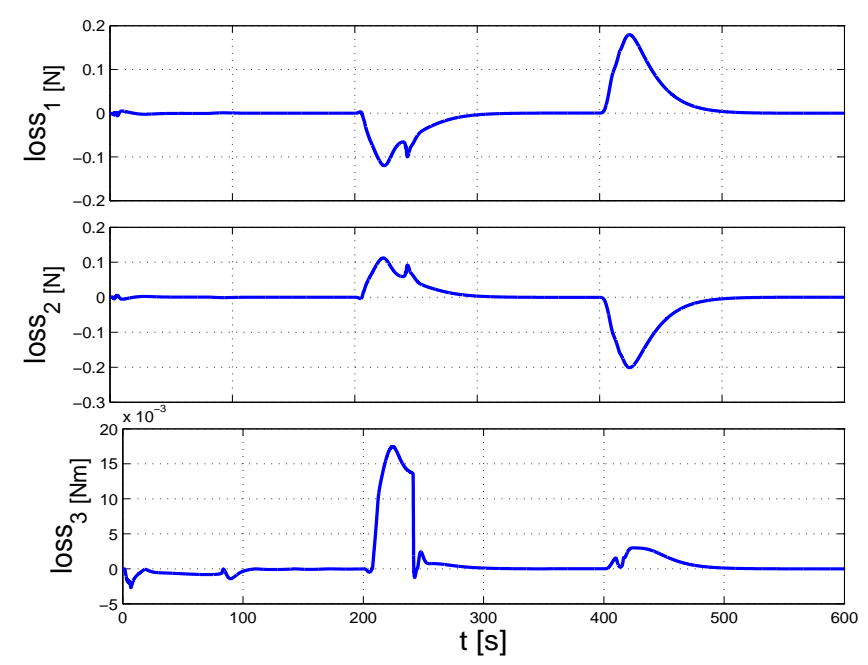

Figure 8: Actual thrust loss

\section{References}

Bodson, M. Evaluation of optimization methods for control allocation. J. Guidance, Control and Dynamics, 2002. 25:703-711.

Buffington, J. M., Enns, D. F., and Teel, A. R. Control allocation and zero dynamics. J. Guidance, Control and Dynamics, 1998. 21:458-464.

Enns, D. Control allocation approaches. In Proc. AIAA Guidance, Navigation and Control Conference and Exhibit, Boston MA. 1998 pages 98-108.

Fossen, T. I. and Blanke, M. Nonlinear output feedback control of underwater vehicle propellers using feedback form estimated axial flow velocity. IEEE Journal of Oceanic Engineering, 2000. 25(2):241255.

Härkegård, O. Efficient active set algorithms for solving constrained least squares problems in aircraft control allocation. In Proc. IEEE Conf. Decision and Control, Las Vegas NV. 2002 .

Johansen, T. A. Optimizing nonlinear control allocation. Proc. IEEE Conf. Decision and Control. Bahamas, 2004. pages 3435-3440.

Johansen, T. A., Fossen, T. I., and Berge, S. P. Constrained nonlinear control allocation with singularity avoidance using sequential quadratic programming. IEEE Trans. Control Systems Technology, 2004. 12:211-216.

Johansen, T. A., Fossen, T. I., and Tøndel, P. Efficient optimal constrained control allocation via multipara- metric programming. AIAA J. Guidance, Control and Dynamics, 2005. 28:506-515.

Lin, Y., Sontag, E. D., and Wang, Y. A smooth converse lyapunov theorem for robust stability. SIAM Journal on Control and Optimization, 1996. 34:124160 .

Lindegaard, K. P. and Fossen, T. I. Fuel-efficient rudder and propeller control allocation for marine craft: Experiments with a model ship. IEEE Trans. Control Systems Technology, 2003. 11:850-862.

Luo, Y., Serrani, A., Yurkovich, S., Doman, D., and Oppenheimer, M. Model predictive dynamic control allocation with actuator dynamics. In Proceedings of the 2004 American Control Conference, Boston, $M A, 2004$.

Luo, Y., Serrani, A., Yurkovich, S., Doman, D., and Oppenheimer, M. Dynamic control allocation with asymptotic tracking of time-varying control trajectories. In Proceedings of the 2005 American Control Conference, Portland, OR, 2005.

Pivano, L., Johansen, T. A., Smogeli, Ø. N., and Fossen, T. I. Nonlinear Thrust Controller for Marine Propellers in Four-Quadrant Operations. American Control Conference (ACC), New York, USA, 2007.

Pivano, L., Smogeli, Ø. N., Johansen, T. A., and Fossen, T. I. Marine propeller thrust estimation in fourquadrant operations. 45th IEEE Conference on Decision and Control, San Diego, CA, USA, 2006.

Poonamallee, V., Yurkovich, S., Serrani, A., Doman, D., and Oppenheimer, M. Dynamic control allocation with asymptotic tracking of time-varying control trajectories. In Proceedings of the 2004 American Control Conference, Boston, MA, 2005.

Sørdalen, O. J. Optimal thrust allocation for marine vessels. Control Engineering Practice, 1997. 5:12231231.

Sørensen, A. J., Ådnanes, A. K., Fossen, T. I., and Strand, J. P. A new method of thruster control in positioning of ships based on power control. Proc. 4 th IFAC Conf. Manoeuvering and Control of Marine Craft, Brijuni, Croatia, 1997.

Teel, A., Panteley, E., and Loria, A. Integral characterization of uniform asymptotic and exponential stability with applications. Maths. Control Signals and Systems, 2002. 15:177-201.

Tjønnås, J., Chaillet, A., Panteley, E., and Johansen, T. A. Cascade lemma for set-stabile systems. 45th 
IEEE Conference on Decision and Control, San Diego, CA, 2006.

Tjønnås, J. and Johansen, T. A. Adaptive optimizing nonlinear control allocation. In Proc. of the 16th IFAC World Congress, Prague, Czech Republic, 2005.

Tjønnås, J. and Johansen, T. A. On optimizing nonlinear adaptive control allocation with actuator dynamics. 7th IFAC Symposium on Nonlinear Control Systems, Pretoria, South Africa, 2007. 